\title{
A Discussion on the Properties of Gamow States
}

\section{Gadella}

\section{Foundations of Physics}

An International Journal Devoted to the Conceptual Bases and Fundamental Theories of Modern Physics

\section{ISSN 0015-9018}

Volume 45

Number 2

Found Phys (2015) 45:177-197

DOI 10.1007/s10701-014-9860-7

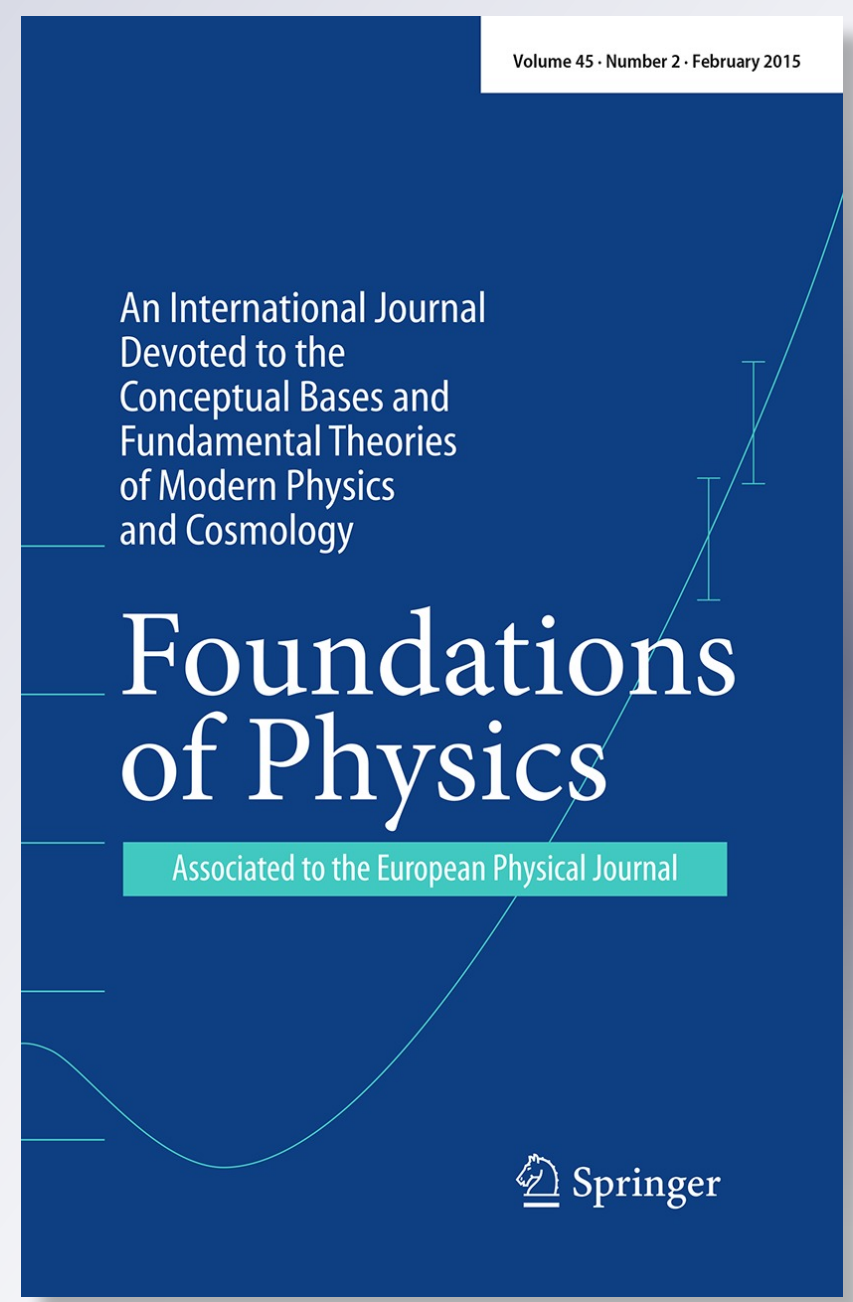

Springer 
Your article is protected by copyright and all rights are held exclusively by Springer Science +Business Media New York. This e-offprint is for personal use only and shall not be selfarchived in electronic repositories. If you wish to self-archive your article, please use the accepted manuscript version for posting on your own website. You may further deposit the accepted manuscript version in any repository, provided it is only made publicly available 12 months after official publication or later and provided acknowledgement is given to the original source of publication and a link is inserted to the published article on Springer's website. The link must be accompanied by the following text: "The final publication is available at link.springer.com". 


\title{
A Discussion on the Properties of Gamow States
}

\author{
M. Gadella
}

Received: 23 June 2014 / Accepted: 28 November 2014 / Published online: 11 December 2014

(C) Springer Science+Business Media New York 2014

\begin{abstract}
Gamow states are vector states for the pure decaying part of a quantum resonance. We review and analyze the properties of Gamow vectors in different representations. In particular, we discuss the controversial problem of assigning a mean value of the energy for a Gamow state from several points of view. The question on whether a Gamow state is a pure state or not is also analyzed here, as has relevance on the assignation of a non-zero value for the entropy for a Gamow state.
\end{abstract}

Keywords Quantum resonances · Gamow states · Energy averages on Gamow states

\section{Introduction}

As is well known, the decay law of a quantum resonance or, equivalently, a quantum decaying state is approximately exponential for almost all times within the interval of observation. Only for very short or very large times, it is possible to detect deviations from this exponential behavior [1-3]. For those times for which the exponential decay dominates, it is often quite convenient to assign to the decaying system a vector state called the Gamow state or Gamow vector, with the property of having an exponential decay at all times after an origin of times that we identify with $t=0$. We shall denote the Gamow vector as $\psi^{D}$ ( $D$ meaning decaying).

However, this creates some formal difficulties. To begin with, an exponential decay law means that the non-decay probability $\mathcal{P}(t):=\left|\left\langle\psi^{D}\left|e^{-i t H}\right| \psi^{D}\right\rangle\right|^{2}$ is an exponential of the type $e^{-\Gamma t}$, for $t \geq 0$ and $\Gamma>0$. Then, the non-decay amplitude should be of the form:

M. Gadella $(\varangle)$

Departamento de Física Teórica, Atómica y Óptica, Universidad de Valladolid,

Paseo Belén 7, 47011 Valladolid, Spain

e-mail: manuelgadella1@gmail.com 


$$
\left\langle\psi^{D}\left|e^{-i t H}\right| \psi^{D}\right\rangle=e^{i \varphi(t)} e^{-\Gamma t / 2}\left\langle\psi^{D} \mid \psi^{D}\right\rangle=e^{i \varphi(t)} e^{-\Gamma t / 2},
$$

where $e^{i \varphi(t)}$ is a time dependent phase. For the moment, we may assume $\psi^{D}$ be normalized to one. Then, compute formally the derivative of (1) with respect to time at $t=0$. The result is

$$
\left.\frac{d}{d t}\left\langle\psi^{D}\left|e^{-i t H}\right| \psi^{D}\right\rangle\right|_{t=0}=-i\left\langle\psi^{D}|H| \psi^{D}\right\rangle=i \varphi^{\prime}(0)-\Gamma,
$$

or

$$
\left\langle\psi^{D}|H| \psi^{D}\right\rangle=-\varphi^{\prime}(0)-i \Gamma .
$$

Therefore the average of the Hamiltonian $H$ on the Gamow state $\psi^{D}$ is a complex number, which is non-sense because $H$ is self adjoint. In this spirit, Nakanishi [4] defined the Gamow vector as an eigenvector of the total Hamiltonian $H=H_{0}+V$ with complex eigenvalue: $H \psi^{D}=\left(E_{R}-i \Gamma / 2\right) \psi^{D}$. Here, $E_{R}$ is the resonance energy and $\Gamma$ the width. In this case, the formal time evolution of $\psi^{D}$ would be $e^{-i t H} \psi^{D}=$ $e^{-i t E_{R}} e^{-t \Gamma / 2} \psi^{D}$, which contradicts the fact that $e^{-i t H}$ is unitary, i.e., that $H$ is self adjoint. Then, the non-decay probability is $e^{-t \Gamma / 2}$, i.e., a decaying exponential.

This description looks somehow convenient, but at the same time it is definitively wrong in the context of a description of pure states as normalizable vectors in a Hilbert space. As a linear operator on a Hilbert space, a self adjoint Hamiltonian cannot have complex eigenvalues. Fortunately, this is not the end of the story. If we enlarge the space of states out of the Hilbert space, we may find that the extended Hamiltonian had complex eigenvalues with well defined eigenvectors.

The mechanism to find complex eigenvalues for self adjoint Hamiltonians is well known and is based in the concept of Gelfand triplet or rigged Hilbert space (RHS). In brief, a RHS is a tern of spaces [5-9]:

$$
\Phi \subset \mathcal{H} \subset \Phi^{\times},
$$

where (i) $\mathcal{H}$ is the Hilbert space of states of the quantum system under consideration. The Hamiltonian $H$ is a self adjoint operator on $\mathcal{H}$. (ii) $\Phi$ is a dense subspace in $\mathcal{H}$ contained in the domain of $H$ and endowed with a topology stronger than the Hilbert space topology. (iii) $\Phi^{\times}$is the space of all continuous antilinear (in order to keep the Dirac notation in use) mappings (functionals) on $\Phi$, with values in the complex plane $\mathbb{C}$. The action of $F \in \Phi^{\times}$on $\varphi \in \Phi$ is a complex number that we denote as $\langle\varphi \mid F\rangle$.

The extension of $H$ into $\Phi^{\times}$is given by the following duality formula: If $\varphi \in \Phi$, $F \in \Phi^{\times}:$

$$
\langle H \varphi \mid F\rangle=\langle\varphi \mid H F\rangle .
$$

Here, we shall also denote by $H$ the extension of $H$ for simplicity. The choice of the space $\Phi$ is not unique and henceforth $\Phi^{\times}$is not unique either. The extension of $H$ into $\Phi^{\times}$may have solutions of the eigenvalue problem with complex eigenvalues, because the restriction of self adjointness does not apply there. Some constructions allow the 
extension of the time evolution operator $e^{-i t H}$ into $\Phi^{\times}$, either for all $t \in \mathbb{R}$ or for $t \geq 0$ [10-12]. In these constructions, formulas like $e^{-i t H} \psi^{D}=e^{-i t E_{R}} e^{-t \Gamma / 2} \psi^{D}$ make sense.

In passing, we should recall that if the non-decay probability of a vector state $\psi$, defined as $\mathcal{P}(t)=\left|\left\langle\psi\left|e^{-i t H}\right| \psi\right\rangle\right|^{2}$, is an exact exponential of the type $e^{-t \Gamma}$, then, its energy distribution (essentially the square of the modulus of the wave function in the energy representation $\psi(E))$ must have the Breit-Wigner form, $|\psi(E)|^{2} \propto$ $\left[\left(E-E_{R}\right)^{2}+\Gamma^{2} / 4\right]^{-1}$ for all real values of $E$, where the sign $\propto$ means "proportional to". This is not possible if $\psi$ is a vector in the Hilbert space of pure states, since the Hamiltonian is semibounded. This means that $\psi(E)$ should be zero below a certain threshold, usually taken equal to zero [13].

Now, the point is that $\psi^{D}$ is usually not a normalizable vector, so that expressions like $\left\langle\psi^{D} \mid \psi^{D}\right\rangle=1$ or worse $\left\langle\psi^{D}|A| \psi^{D}\right\rangle$, where $A$ is the Hamiltonian or any other observable, do not make sense in general.

Several attempts have been made in order to propose either an expression for $\left\langle\psi^{D} \mid \psi^{D}\right\rangle$ [14-16], or for $\left\langle\psi^{D}|A| \psi^{D}\right\rangle$ [17-19]. The definition by Berggren [17,18] is assumed to be valid for any observable $A$ and not only for the Hamiltonian. Here, we focus our attention on the average of the Hamiltonian on a Gamow vector, for which four different results have been proposed:

- This idea was discussed in [4] or in [20]. If $H\left|\psi^{D}\right\rangle=\left(E_{R}-i \Gamma / 2\right)\left|\psi^{D}\right\rangle$ and $\left\langle\psi^{D}\right| H=\left(E_{R}+i \Gamma / 2\right)\left\langle\psi^{D}\right|$, then, $\left\langle\psi^{D}|H| \psi^{D}\right\rangle=\left(E_{R}-i \Gamma / 2\right)\left\langle\psi^{D} \mid \psi^{D}\right\rangle=$ $\left(E_{R}+i \Gamma / 2\right)\left\langle\psi^{D} \mid \psi^{D}\right\rangle$. This would imply that $\left\langle\psi^{D}|H| \psi^{D}\right\rangle=0$. Again, the difficulty lies on the ill definition of $\left\langle\psi^{D} \mid \psi^{D}\right\rangle$.

- Along the decaying Gamow vector, one can define its time reversal, the growing Gamow vector $\psi^{G}$, which is a functional with the following properties: $H \psi^{G}=$ $\left(E_{R}+i \Gamma / 2\right) \psi^{G}$ and $e^{-i H t} \psi^{G}=e^{-i t E_{R}} e^{t \Gamma / 2} \psi^{G}$. In principle, one can define the bracket $\left\langle\psi^{G} \mid \psi^{D}\right\rangle$, which can be normalized to one. We shall show how this can be done within the context of the Friedrichs model later. Then, $\left\langle\psi^{G}|H| \psi^{D}\right\rangle=$ $\left(E_{R}-i \Gamma / 2\right)\left\langle\psi^{G} \mid \psi^{D}\right\rangle=E_{R}-i \Gamma / 2$ may look like a reasonable definition. However, this mean value contains two terms, one is the resonance energy $E_{R}$ and the other $\Gamma$ is the width, which is assumed to be the inverse of the half life time $\tau=\Gamma^{-1}$. Simultaneous measurements of these two values are supposed to be forbidden by the time-energy incertitude principle. Therefore, although well defined, this definition for the mean value of the energy has an uncertain physical meaning.

- The approach $\left\langle\psi^{D}|H| \psi^{D}\right\rangle=E_{R}$ is largely discussed in [19].

- According to the Berggren definition [17,18], one has $\left\langle\psi^{D}|H| \psi^{D}\right\rangle=E_{R}+o\left(\Gamma^{2}\right)$. Note that these two latter proposals coincide at order zero on $\Gamma$. This one can be generalized to other observables [17-19]. See a discussion on this approach in [21].

\subsection{Motivation and Organization}

Resonances occur when the analytic continuation of the $S$-operator shows poles with non-vanishing imaginary parts. In the energy representation, these complex poles 
appear in complex conjugate pairs of the same multiplicity. Along the present paper, we are always assuming that this multiplicity is equal to one for simplicity. A definition of resonances in terms of the analytic continuation of the resolvent of the total Hamiltonian is also possible [22,23]. Although not always [1], these two definitions are usually equivalent. This is the case for the Friedrichs model, which we shall briefly describe in Sect. 2.

In the first paragraph of the present Introduction, we have mentioned that deviations from the exponential decay law have been detected only for very short or very large times, as compared with the mean life. From the theoretical point of view, this happens whenever the decaying state is represented by a normalizable vector in Hilbert space $\psi[1]$. It is always possible to split this vector space into a sum of two contributions $\psi=\psi^{D}+\psi^{B}$, where $\psi^{D}$ is the decaying Gamow vector and $\psi^{B}$ is a background term, which are supposed to be responsible for these deviations. In principle, the vector state $\psi$ can be always chosen in some way so that $\psi^{B}$ be small in some sense, and if this were the case, the main contribution to the vector state $\psi$ is given by its "Gamow part", $\psi^{D}$.

Most of the period of observation belongs to those intermediate times for which the decay is approximately exponential. Then, the use of $\psi^{D}$ as vector state is a good approximation. We should be able to evaluate observable averages on Gamow states, or at least to have a way to propose definitions for these averages. The most obvious case, as we have discussed earlier is the energy average values. As an intermediate step, we need some well posed definition of scalar products between Gamow vectors or between a Gamow vector with itself.

In order to understand the origin of these difficulties better, we recall the structure of the Gamow vectors for the simplest Friedrichs model. In this case, scalar products (that should be more properly called brackets) of the form $\left\langle\psi^{D} \mid \psi^{D}\right\rangle$ or $\left\langle\psi^{G} \mid \psi^{G}\right\rangle$ cannot be constructed with the usual machinery of the model. This is due to the fact that the explicit expression for the Gamow vectors contains distributional terms that cannot be multiplied and come from the explicit form of the background. Since the effect of the background is small over large time intervals, it may be convenient to stay in the space spanned by Gamow vectors and get rid of the background contribution.

Finally, we address to the following question: Are Gamow states pure states? Since they are expressed as vector states and not as densities, a naive answer would have been yes. Pure states have zero entropy. On the other hand, Gamow states decay (either to the future like $\psi^{D}$ or to the past like $\psi^{G}$ ) and decay exponentially. This may suggest that the entropy of a Gamow state cannot be zero. A non-zero entropy for decaying states was suggested in [20]. With the use of an algebraic formalism developed by Antoniou et al. [24] in order to study the so called singular diagonal states [25-27], we show that Gamow states cannot be pure states. In this context, Gamow states can be expressed as functionals over an algebra, at least in three different forms. Each of these forms gives different averages for the energy of Gamow vectors and all these averages have been already discussed in the literature.

This paper is organized into this Introduction and other five sections as follows: In Sect. 2, we discuss the difficulties to define brackets (or scalar products) among Gamow vectors in the Friedrichs model. We see that in some cases this is possible while in others do not. 
In Sect.3, we get rid of the background and work in spaces spanned by Gamow vectors. In this case, it is trivial to define scalar products and other brackets between vectors of these spaces. Their interest lies in the possibility of proposing well defined energy averages for some of the possibilities discussed in the literature.

The explicit construction of normalizable vectors in the energy representation that approximates Gamow vectors is made in Sect. 4, where we discuss the meaning of this approximation.

Finally, in Sect. 5 we show that a Gamow vector can be given as a functional over an algebra of observables [24,28,29]. We show that this can be done, at least, in three different forms and this is totally new. Each one of these forms give different values for the energy averages. None of these three possible functionals representing a Gamow state is a pure state.

The paper is closed with a section on concluding remarks.

\section{The Friedrichs Model and More}

It is interesting the use of the Friedrichs model in order to understand the difficulties for a rigorous definition of brackets between Gamow vectors. In fact, the Friedrichs model is the first and the simplest rigorous model for quantum decay phenomena. It is much more than a toy model, since it serves as to appreciate the basic features of quantum decay. The basic Friedrichs model and its successive refinements serve as general models for both relativistic and non-relativistic quantum decay [12,22,30,31]. In order to make this article self contained, let us recall the basic features of the simplest Friedrichs model.

A quantum model with resonances, has two Hamiltonians, a free Hamiltonian $H_{0}$ and a total or perturbed Hamiltonian $H=H_{0}+V$, where $V$ is a potential which is the interaction responsible for the production of resonances. In the case of the simplest Friedrichs model, $H_{0}$ has a non-degenerate continuous spectrum from 0 to $\infty$ and one bound state embedded in the continuous spectrum. The potential $V$ intertwines the discrete and the continuous spectrum of $H_{0}$. As a consequence, the bound state of $H_{0}$ becomes unstable. Explicitely,

$$
H_{0}=\omega_{0}|1\rangle\left\langle 1\left|+\int_{0}^{\infty} d \omega \omega\right| \omega\right\rangle\left\langle\omega\left|, \quad H_{0}\right| 1\right\rangle=\omega_{0}|1\rangle, \quad H_{0}|\omega\rangle=\omega|\omega\rangle,
$$

with $\omega_{0}>0$ and $\omega \in[0, \infty)$. The potential $V$ is

$$
V=\int_{0}^{\infty} f(\omega)[|1\rangle\langle\omega|+| \omega\rangle\langle 1|] d \omega
$$

where $f(\omega)$ is square integrable and can be chosen real without loss of generality. It is called the form factor. In order that the forthcoming discussion be valid, we need some extra mathematical assumptions on the form factor such as $f\left(\omega_{0}\right) \neq 0$ and that the function $f^{2}(\omega)$ be analytically continuable on an open set containing the positive semiaxis $\mathbb{R}^{+} \equiv[0, \infty)$. 
The total Hamiltonian is $H=H_{0}+\lambda V$, where $\lambda$ is a real constant (usually small so that perturbation theory can be applied) called the coupling constant. The total Hamiltonian $H$ has purely absolutely continuous spectrum for $\lambda>0$, which is equal to $\mathbb{R}^{+}$and has no real eigenvalues.

As a result of the interaction, the bound state $|1\rangle$ of $H_{0}$ becomes unstable. Today, it is commonly accepted that unstable quantum states and quantum resonances are the same physical objects, so that we shall refer to both indistinctly. A discussion on this can be found for instance in [32,33]. Resonances are usually obtained as poles of the analytic continuation on the complex plane of a multivalued function called the reduced resolvent, which is:

$$
\varphi(z):=\left\langle 1\left|\frac{1}{H-z}\right| 1\right\rangle
$$

This function is analytic on the entire complex plane, except for a branch cut on the positive real semiaxis $\mathbb{R}^{+} \equiv[0, \infty)$. It admits analytic continuations through the cut from above to below (i.e., from the upper half plane into the lower half plane) and from below to above (from the lower into the upper). These analytic continuations cannot coincide with the values that $f(z)$ has on these half planes, so that it is often convenient the use of a two sheeted Riemann surface to support the values for $\varphi(z)$.

Under the conditions stated before, the analytic continuation from above to below of the function $\varphi(w)$ has a simple pole at the point given by the following integral equation $[22,34]$ :

$$
z_{R}=\omega_{0}+\int_{0}^{\infty} d \omega \frac{\lambda^{2} f^{2}(\omega)}{\left[z_{R}-\omega\right]_{+}}:=\omega_{0}+\int_{0}^{\infty} d \omega \frac{\lambda^{2} f^{2}(\omega)}{z_{R}-\omega}-2 \pi i \lambda^{2}\left|f\left(z_{R}\right)\right|^{2}
$$

Let us recall that we have assumed that $f^{2}(\omega)$ is analytically continuable, so that $\left|f\left(z_{R}\right)\right|^{2}$ is well defined. In addition the analytic continuation of $\varphi(\omega)$ from below to above has a pole at $z_{R}^{*}$, where the asterisk denotes complex conjugation, so that $\left|f\left(z_{R}^{*}\right)\right|^{2}$ is also well defined. The Gamow vectors $\psi^{D}$ and $\psi^{G}$ are obtained by the following formulas:

$$
\begin{aligned}
& \left|\psi^{D}\right\rangle=N\left[|1\rangle+\int_{0}^{\infty} d \omega \frac{\lambda f(\omega)}{\left[z_{R}-\omega\right]_{+}}|\omega\rangle\right] \\
& \left|\psi^{G}\right\rangle=N\left[|1\rangle+\int_{0}^{\infty} d \omega \frac{\lambda f(\omega)}{\left[z_{R}^{*}-\omega\right]_{-}}|\omega\rangle\right],
\end{aligned}
$$

where $N$ is a normalization constant, which is assigned by convenience. These vectors are functionals on some test function spaces, as discussed in several articles [12,31,34]. If we call $\Phi_{-}$and $\Phi_{+}$, respectively, the test vector space for $\psi^{D}$ and $\psi^{G}$ and $\phi_{ \pm} \in \Phi_{ \pm}$, the meaning of (10) and (11) is:

$$
\left\langle\phi_{-} \mid \psi^{D}\right\rangle=N\left[\left\langle\phi_{-} \mid 1\right\rangle+\int_{0}^{\infty} d \omega \frac{\lambda f(\omega)}{\left[z_{R}-\omega\right]_{+}}\left\langle\phi_{-} \mid \omega\right\rangle\right]
$$




$$
\left\langle\phi_{+} \mid \psi^{G}\right\rangle=N\left[\left\langle\phi_{+} \mid 1\right\rangle+\int_{0}^{\infty} d \omega \frac{\lambda f(\omega)}{\left[z_{R}^{*}-\omega\right]_{-}} \mid\left\langle\phi_{+} \mid \omega\right\rangle\right] .
$$

Note that $\left\langle\phi_{-} \mid \omega\right\rangle$ and $\left\langle\phi_{+} \mid \omega\right\rangle$ are functions of $\omega \in \mathbb{R}^{+}$. The products $\left\langle\phi_{-} \mid 1\right\rangle$ and $\left\langle\phi_{+} \mid 1\right\rangle$ are well defined as scalar products on Hilbert space. Then, the meaning in the plus sign in the denominator of (12) has been explained in (9). The minus sign on the denominator of (13) has a similar meaning.

Unfortunately, there is no way to define $\left\langle\psi^{D} \mid \psi^{D}\right\rangle$ or $\left\langle\psi^{G} \mid \psi^{G}\right\rangle$ using the expressions (10) and (11) along with $\left\langle\omega \mid \omega^{\prime}\right\rangle=\delta\left(\omega-\omega^{\prime}\right)$ and $\langle 1 \mid \omega\rangle=0$. Take for instance $\left\langle\psi^{D} \mid \psi^{D}\right\rangle$. According to (10), we should have

$$
\left\langle\psi^{D} \mid \psi^{D}\right\rangle=N^{2}\left[1+\int_{0}^{\infty} d \omega \frac{\lambda^{2} f^{2}(\omega)}{\left[z_{R}^{*}-\omega\right]_{-}\left[z_{R}-\omega\right]_{+}}\right] .
$$

The integral in (14) denotes the action on the function $f^{2}(\omega)$ of the product of two distributions $1 /\left[z_{R}^{*}-\omega\right]_{-}$and $1 /\left[z_{R}-\omega\right]_{+}$and this product is not well defined in general. Thus, an expression like $\left\langle\psi^{D} \mid \psi^{D}\right\rangle$ cannot be properly defined in the mere framework of the Friedrichs model. Same can be said about $\left\langle\psi^{G} \mid \psi^{G}\right\rangle$.

However as mentioned earlier, expressions like $\left\langle\psi^{D} \mid \psi^{G}\right\rangle$ and $\left\langle\psi^{G} \mid \psi^{D}\right\rangle$ can be well defined by using (10) and (11) and their conjugates (for details see [35]). This gives:

$$
\left\langle\psi^{D} \mid \psi^{G}\right\rangle=N^{2}\left[1+\int_{0}^{\infty} d \omega \frac{\lambda^{2} f^{2}(\omega)}{\left[z_{R}^{*}-\omega\right]_{-}^{2}}\right],
$$

and a similar expression for $\left\langle\psi^{G} \mid \psi^{D}\right\rangle$ (replace the denominator of the function under the integral by $\left.\left[z_{R}-\omega\right]_{+}^{2}\right)$. The integral in (15) is well defined as is the action on $f(\omega)$ of the derivative of the distribution $1 /\left[z_{R}^{*}-\omega\right]_{-}$(or $1 /\left[z_{R}-\omega\right]_{+}$if we had $\left.\left\langle\psi^{G} \mid \psi^{D}\right\rangle\right)$, in the usual distributional sense. Then, the constant $N^{2}$ can be determined so that $\left\langle\psi^{D} \mid \psi^{G}\right\rangle=1=\left\langle\psi^{G} \mid \psi^{D}\right\rangle$.

We can also construct a Friedrichs model with a finite number, $N$, of resonances $[31,36]$. In this case, the total Hamiltonian is $H=H_{0}+\lambda V$ with:

$$
\begin{aligned}
H_{0} & :=\sum_{i=1}^{N} \omega_{i}|i\rangle\left\langle i\left|+\int_{0}^{\infty} \omega\right| \omega\right\rangle\langle\omega| d \omega, \\
V & :=\sum_{i=1}^{N} \int_{0}^{\infty} f_{i}(\omega)[|\omega\rangle\langle i|+| i\rangle\langle\omega|] d \omega,
\end{aligned}
$$

where the form factor functions $\left\{f_{i}(\omega)\right\}$ are square integrable on $[0, \infty)$ and analytically continuable, just as $f(\omega)$. The eigenvalues $\omega_{1}, \ldots, \omega_{N}$ of $H_{0}$ are assumed to be positive. Furthermore, if $i \neq j$, one also assumes that $|i\rangle \neq|j\rangle$, so that the energy levels of $H_{0}$ are non-degenerate. This assumption is made for simplicity only. As in the case of only one eigenvalue, when the potential $\lambda V$ is switched on, the eigenvalues of $H_{0}$ are transformed into resonances. Let us call $z_{1}, z_{2}, \ldots, z_{N}$ to the respective resonance poles. Their corresponding decaying Gamow vectors have the form: 


$$
\left|\psi_{i}^{D}\right\rangle=|i\rangle+\int_{0}^{\infty} d \omega \frac{\lambda f(\omega)}{\left[z_{i}-\omega\right]_{+}}|\omega\rangle, \quad i=1,2, \ldots, N,
$$

and a similar equation for the growing Gamow vectors, $\left|\psi_{i}^{G}\right\rangle, i=1,2, \ldots, N$. We note that, in this context, not only $\left\langle\psi_{i}^{D} \mid \psi_{i}^{D}\right\rangle, i=1,2, \ldots, N$, but even expressions like $\left\langle\psi_{i}^{D} \mid \psi_{j}^{D}\right\rangle, i \neq j$, etc are not defined.

Leaving aside the Friedrichs model, for some other quantum models we may find a wave function valid for unstable states. This wave function plays the role of Gamow vector. This is the case when $H_{0}$ is the Hamiltonian for the free particle and $H=$ $H_{0}+V$, where $V=V(\rho)$ is a spherically symmetric potential of short range. In this case, wave functions for unstable states have a radial part that behaves asymptotically as $e^{\kappa \rho}$, where $\rho$ is the distance to the center of forces and $\kappa>0$. In this case, some brackets among Gamow states have been defined. Here, the basic tool is the well known Zeldovich regularization method [37]. See details in [14,15] and another point of view in [38].

\section{The Space of Gamow Vectors}

So far, we have seen some of the difficulties arisen when we try to define brackets among Gamow vectors. The situation looks like worse when we note that, even in the cases in which regularized brackets can be defined, the meaning of energy averages like $\left\langle\psi^{D}|H| \psi^{D}\right\rangle$ or $\left\langle\psi^{G}|H| \psi^{G}\right\rangle$ is not clear.

From our experience with the Friedrichs model, it seems that these difficulties may come from the consideration of quantum decaying states as open systems where the background (consequence of a series of effects responsible for the deviations of the pure exponential decay law) plays a substantial role. However, we have already mentioned that, for large periods of experimentation, the decay is (approximately) exponential. Thus suggest that the assumption that the set of decaying states forms a closed system is not a bad approximation. At least for times neither too short or too large.

This idea drastically changes the scenario, as it allows us to consider the space spanned by the Gamow vectors as the truly space of states for a system in which our solely interest is focused into these unstable quantum states.

Our experience shows that it is always possible the separation between the Gamow state and the background [10-12]. Then, let $\psi$ be a quantum (normalizable) state, such that its survival probability $\mathcal{P}(t)=\left|\left\langle\psi\left|e^{-i t H}\right| \psi\right\rangle\right|^{2}$ is, approximately with a good degree of accuracy, a decaying exponential for almost all values of $t>0$ (with exception of $t \approx 0$ or $t$ large). Then, one can decompose $\psi$ as

$$
\psi=\sum_{i} a_{i} \psi_{i}^{D}+\psi^{B}
$$

where $\left\{\psi_{i}^{D}\right\}$ are the decaying Gamow vectors, and $\psi^{B}$ the (non-normalizable) quantum state vector for the background. Along to this decomposition, there is another one that makes use of the growing Gamow vectors $\psi_{i}^{G}[11,12]$. Here we have that $H \psi_{i}^{D}=$ 
$z_{i} \psi_{i}^{D}$ and $H \psi_{i}^{G}=z_{i}^{*} \psi_{i}^{G}$, where $z_{i}$ and $z_{i}^{*}$ are the pair of poles of the analytic continuation of the $S$-matrix, $S(E)$, characterizing the $i$-th resonance. Gamow vectors $\psi_{i}^{D}$ and $\psi_{i}^{G}$ belong to a convenient space of functionals $[11,12]$.

To understand the situation better, let us assume that we have only one resonance in the sum (18), so that it has the form $\psi=a \psi^{D}+\psi^{B}$. Then, the non-decay amplitude is now:

$$
\begin{aligned}
\left\langle\psi\left|e^{-i t H}\right| \psi\right\rangle & =\left\langle\psi \left| e^{-i t H}\left(a\left|\psi^{D}\right\rangle+\left|\psi^{B}\right\rangle\right)\right.\right. \\
& =a\left\langle\psi\left|e^{-i t H}\right| \psi^{D}\right\rangle+\left\langle\psi\left|e^{-i t H}\right| \psi^{B}\right\rangle \\
& =a e^{-i t E_{R}} e^{-\Gamma t / 2}\left\langle\psi \mid \psi^{D}\right\rangle+\left\langle\psi\left|e^{-i t H}\right| \psi^{B}\right\rangle .
\end{aligned}
$$

This formula shows that the background is responsible for all possible deviations of the exponential decay.

A technical point: Since $\psi^{D}$ is not a vector in Hilbert space, but a functional on a space of test vectors, the question is if $\left\langle\psi \mid \psi^{D}\right\rangle$ is well defined for any normalizable $\psi$. The answer is: No. However, since the space of test vectors, in which $\psi^{D}$ acts, is dense in the total Hilbert space, the error of replacing $\psi$ by a test vector can be arbitrarily small.

Then, within the large time interval in which exponential decay (or oscillating decay for the case of more than one resonance) is dominant, we may drop the background and consider the effect of resonance poles only. The oscillating decay would be an effect of interference between resonances [36].

Along the decomposition (19), we have a spectral decomposition for the total Hamiltonian $H$, which can be written as:

$$
H=\sum_{i} z_{i}\left|\psi_{i}^{D}\right\rangle\left\langle\psi_{i}^{G}\right|+B P
$$

where $B P$ stands for "background part". Its explicit form is irrelevant as we are only interested in the Gamow contribution, given by the sum in (20), and therefore we shall drop $B P$ out.

It is at this point when we start considering the space of unstable quantum states as a closed system.

Note that in the expression (20) there is no trace of a property like Hermiticity. As a matter of fact, the formal Hermitian conjugate of (20), where we drop the background term, is given by:

$$
H^{\dagger}=\sum_{i} z_{i}^{*}\left|\psi_{i}^{G}\right\rangle\left\langle\psi_{i}^{D}\right|
$$

In the general study on the construction of Gamow vectors, we made use of two Gelfand triples, $\Phi_{ \pm} \subset \mathcal{H} \subset \Phi_{ \pm}^{\times}[11,12]$, so that $\psi_{i}^{D} \in \Phi_{+}^{\times}$and $\psi_{i}^{G} \in \Phi_{-}^{\times}$, for all $i$. Therefore, $\psi_{i}^{D}$ are functionals on $\Phi_{+}$and $\psi_{i}^{G}$ are functionals on $\Phi_{-}$. Then, note that $H$ as in (20) acts on vectors in $\Phi_{+}$and transforms them in vectors in $\Phi_{-}^{\times}$and $H^{\dagger}$ as in (21) acts on vectors of $\Phi_{-}$and transforms them on vectors in $\Phi_{+}^{\times}$. Thus for any $\varphi_{ \pm} \in \Phi_{ \pm}$, we have: 


$$
H \varphi_{-}=\sum_{i} z_{i}\left|\psi_{i}^{D}\right\rangle\left\langle\psi_{i}^{G} \mid \varphi_{-}\right\rangle \in \Phi_{+}^{\times}, \quad H^{\dagger} \varphi_{+}=\sum_{i} z_{i}^{*}\left|\psi_{i}^{G}\right\rangle\left\langle\psi_{i}^{D} \mid \varphi_{+}\right\rangle \in \Phi_{-}^{\times}
$$

This shows that $H$ and $H^{\dagger}$ must belong to different spaces. If we call $\mathcal{L}(\Phi, \Psi)$ to the space of continuous linear functionals from the topological vector space $\Phi$ into the topological vector space $\Psi$, we conclude that [39]

$$
H \in \mathcal{L}\left(\Phi_{-}, \Phi_{+}^{\times}\right) ; \quad H^{\dagger} \in \mathcal{L}\left(\Phi_{+}, \Phi_{-}^{\times}\right),
$$

so that they belong to different spaces. For our purposes, we would like to have a formally Hermitian Hamiltonian. Its construction is simple and we shall do it in the sequel.

To this end, let us consider the subspace $\mathcal{H}^{D}$ spanned by the Gamow vectors $\psi_{i}^{D}$, in one side, and $\mathcal{H}^{G}$ spanned by $\psi_{i}^{G}$ in the other, $i=1,2, \ldots, N$. The number $N$ of resonances can be either finite or infinite, but in the studied case of multiple resonance Friedrichs model, $N$ is finite. However, in realistic models in non-relativistic quantum mechanics, the consideration of an infinite number of resonances makes no clear physical sense. Since resonance poles are isolated, two situations may happen (independently or at the same time):

(i) Either the imaginary part of the resonance poles is, save for a finite number of them, too big. Then, the mean life for these resonances is negligible and they become unobservable.

(ii) Or the real parts of the resonance poles become too large. These real parts are identified with the resonance energies. These resonances are in the relativistic regime, which we have discarded from our analysis.

Thus, we may assume that the number of resonance poles is finite, although this would not really affect to the forthcoming mathematical discussion. Either finite or infinite dimensional, we may always assume that $\left\{\psi_{i}^{D}\right\}$ and $\left\{\psi_{i}^{G}\right\}$ form an orthonormal basis for $\mathcal{H}^{D}$ and $\mathcal{H}^{G}$, respectively. This just serves to construct a scalar product in both spaces.

As a state vector for the exponentially decaying part of a resonance, we may use $\psi^{D}$ or $\psi^{G}$ indistinctly. If we want to have a description that includes both types of state vectors, it would be convenient to use the sum of spaces $\mathcal{H}^{D} \oplus \mathcal{H}^{G}$. Is in this spaces, where objects like $\left\langle\psi_{i}^{D} \mid \psi_{j}^{G}\right\rangle$ could make sense.

Because of the form we have defined the scalar product, the identity on the space $\mathcal{H}^{D} \oplus \mathcal{H}^{G}$ is given by:

$$
I=\sum_{i}\left\{\left|\psi_{i}^{D}\right\rangle\left\langle\psi_{i}^{G}|+| \psi_{i}^{G}\right\rangle\left\langle\psi_{i}^{D}\right|\right\}
$$

Note that $\left|\psi_{i}^{D}\right\rangle\left\langle\psi_{i}^{G}\right|$ and $\left|\psi_{i}^{G}\right\rangle\left\langle\psi_{i}^{D}\right|$ are the adjoint of each other. Then, the Hamiltonian (without the background) must have the following spectral decomposition:

$$
H=\sum_{i} z_{i}\left|\psi_{i}^{D}\right\rangle\left\langle\psi_{i}^{G}\left|+\sum_{i} z_{i}^{*}\right| \psi_{i}^{G}\right\rangle\left\langle\psi_{i}^{D}\right|
$$


which is obviously Hermitian. Here, $H \psi_{i}^{D}=z_{i} \psi_{i}^{D}$ and $H \psi_{i}^{G}=z_{i}^{*} \psi_{i}^{G}$. This and the spectral decomposition (24) are compatible if and only if $\left\langle\psi_{i}^{G} \mid \psi_{j}^{D}\right\rangle=\delta_{i j}$ and $\left\langle\psi_{i}^{G} \mid \psi_{j}^{G}\right\rangle=\left\langle\psi_{i}^{D} \mid \psi_{j}^{D}\right\rangle=0$, for all $i, j=1,2, \ldots, N$. Therefore, we should give a meaning to these brackets, a task we shall do in the next separated subsection.

\subsection{The Spaces of Gamow Vectors with a Pseudometric}

Once we have isolated the space of Gamow vectors from the background, we can easily provide of good definitions for the mean value of the energy, as well as for the scalar products or brackets of Gamow vectors, given in [4,20]. For a better understanding, we shall start our discussion with a toy model with only one resonance. In this case, the basis for $\mathcal{H}^{D} \oplus \mathcal{H}^{G}$ is $\left\{\psi^{D}, \psi^{G}\right\}$. We may introduce on $\mathcal{H}^{D} \oplus \mathcal{H}^{G}$ a scalar product such that $\left\{\psi^{D}, \psi^{G}\right\}$ be an orthonormal basis. In addition, we are going to introduce here a pseudometric which will be very convenient for our purposes. Note that $\mathcal{H}^{D} \oplus \mathcal{H}^{G}$ can be realized by $\mathbb{C}^{2}$, the two dimensional complex vector space. In $\mathbb{C}^{2}$, we introduce the pseudometric given by the following Hermitian matrix:

$$
A=\left(\begin{array}{ll}
0 & 1 \\
1 & 0
\end{array}\right)
$$

so that if $\psi, \varphi \in \mathbb{C}^{2}$ with components $\psi=\left(\psi^{1}, \psi^{2}\right), \varphi=\left(\varphi^{1}, \varphi^{2}\right)$, one has the following product of $\psi$ and $\varphi$ :

$$
(\psi \mid \varphi):=\left(\psi^{1}, \psi^{2}\right)\left(\begin{array}{ll}
0 & 1 \\
1 & 0
\end{array}\right)\left(\begin{array}{l}
\varphi^{1} \\
\varphi^{2}
\end{array}\right)=\psi^{1} \varphi^{2}+\psi^{2} \varphi^{1}
$$

Since in the basis $\left\{\psi^{D}, \psi^{G}\right\}$, we have

$$
\psi^{D}=\left(\begin{array}{l}
1 \\
0
\end{array}\right), \quad \psi^{G}=\left(\begin{array}{l}
0 \\
1
\end{array}\right)
$$

then,

$$
\begin{gathered}
\left(\psi^{D} \mid \psi^{G}\right)=\left(\psi^{G} \mid \psi^{D}\right)=1, \\
\left(\psi^{D} \mid \psi^{D}\right)=\left(\psi^{G} \mid \psi^{G}\right)=0 .
\end{gathered}
$$

In the present case, it is convenient to write the Hamiltonian in (25) as:

$$
\left.H=z_{R} \mid \psi^{D}\right)\left(\psi^{G}\left|+z_{R}^{*}\right| \psi^{G}\right)\left(\psi^{D} \mid,\right.
$$

which in matrix form reads:

$$
H=\left(\begin{array}{cc}
0 & z_{R} \\
z_{R}^{*} & 0
\end{array}\right)
$$


With this formalism, it is quite simple to obtain some of the averages often discussed in the literature $[4,19,20]$. We can write our results, either writing the Hamiltonian as in (31) or in the matrix form (32). In fact, with (31) we have:

$$
\begin{aligned}
\left(\psi^{D}|H| \psi^{D}\right) & =\left(\psi^{D}\left|\left\{z_{R} \mid \psi^{D}\right)\left(\psi^{G}\left|+z_{R}^{*}\right| \psi^{G}\right)\left(\psi^{D} \mid\right\}\right| \psi^{D}\right) \\
& =z_{R}\left(\psi^{D} \mid \psi^{D}\right)\left(\psi^{G} \mid \psi^{D}\right)+z_{R}^{*}\left(\psi^{D} \mid \psi^{G}\right)\left(\psi^{D} \mid \psi^{D}\right)=0 .
\end{aligned}
$$

The same expression can be obtained using the matrix form (32). Same procedures give:

$$
\left(\psi^{G}|H| \psi^{G}\right)=0, \quad\left(\psi^{G}|H| \psi^{D}\right)=z_{R}, \quad\left(\psi^{D}|H| \psi^{G}\right)=z_{R}^{*}
$$

This result provides of formal sense to the results given in [4,20].

These results can be trivially generalized to the case of $N$ resonances (even to the case of an infinite number of resonances). In this case, we define the pseudoscalar product $(-\mid-)$ with the matrix

$$
A:=\left(\begin{array}{ccccccc}
0 & 1 & \ldots & \ldots & \ldots & \ldots & \ldots \\
1 & 0 & \ldots & \ldots & \ldots & \ldots & \ldots \\
\ldots & \ldots & 0 & 1 & \ldots & \ldots & \ldots \\
\ldots & \ldots & 1 & 0 & \ldots & \ldots & \ldots \\
\ldots & \ldots & \ldots & \ldots & \ldots & \ldots & \ldots \\
\ldots & \ldots & \ldots & \ldots & \ldots & 0 & 1 \\
\ldots & \ldots & \ldots & \ldots & \ldots & 1 & 0
\end{array}\right)
$$

where all other entries in the matrix (32) are equal to zero. Then, we have:

$$
\begin{aligned}
\left(\psi_{i}^{D} \mid \psi_{j}^{D}\right) & =\left(\psi_{i}^{G} \mid \psi_{j}^{G}\right)=0, \\
\left(\psi_{i}^{G} \mid \psi_{j}^{D}\right) & =\left(\psi_{i}^{D} \mid \psi_{j}^{G}\right)=\delta_{i j} . \\
\left(\psi_{i}^{D}|H| \psi_{j}^{D}\right) & =\left(\psi_{i}^{G}|H| \psi_{j}^{G}\right)=0, \\
\left(\psi_{i}^{G}|H| \psi_{j}^{D}\right) & =z_{i} \delta_{i j}, \quad\left(\psi_{i}^{D}|H| \psi_{j}^{G}\right)=z_{i}^{*} \delta_{i j} \quad i, j=1,2, \ldots, N,
\end{aligned}
$$

where $\delta_{i j}$ is the Kronecker delta. These results are consistent with (34).

\section{Approximate Gamow Vectors}

From a mathematical point of view, there exist normalizable state vectors arbitrarily close, in some sense, to a Gamow vector $[11,12]$. From a physical point of view, an explicit construction of a normalizable state vector with an approximately exponential time decay on a wide interval is also possible [1]. However, explicit constructions of approximations to Gamow states are not easy to find, in general. Based on the energy representation, we here propose a simple way to construct such normalizable 
approximations, where the accuracy of the approximation depends on the resonance energy and life time.

Gamow vectors are often defined as functionals on a space of complex functions analytic at least on a half plane. Let us consider two rigged Hilbert spaces $\Phi_{ \pm} \subset$ $\mathcal{H} \subset \Phi_{ \pm}^{\times}$, where $\Phi_{+}$and $\Phi_{-}$admit a realization as complex analytic functions on the upper and lower half of the complex plane, respectively. We do not want to enter here into details that have been largely explained in the literature $[11,12]$. Let us consider a resonance characterized by the resonance poles $z_{R}=E_{R}-i \Gamma / 2$ and its complex conjugate. Decaying and growing Gamow vectors are functionals on $\Phi_{+}^{\times}$and $\Phi_{-}^{\times}$ respectively and can be written in the following form [40,41]:

$$
\psi^{D}=\sqrt{\frac{\Gamma}{2 \pi}} \int_{-\infty}^{\infty} \frac{\left|E^{+}\right\rangle d E}{\left(E-E_{R}\right)+i \Gamma / 2}, \quad \psi^{G}=\sqrt{\frac{\Gamma}{2 \pi}} \int_{-\infty}^{\infty} \frac{\left|E^{-}\right\rangle d E}{\left(E-E_{R}\right)-i \Gamma / 2},
$$

where $\left|E_{ \pm}\right\rangle \in \Phi_{ \pm}^{\times}$are generalized eigenvectors of the total Hamiltonian $H, H\left|E_{ \pm}\right\rangle=$ $E\left|E_{ \pm}\right\rangle$, for all real values of $E$ (for simplicity, we have assumed that the energy spectrum is simple, as is in the case of the Friedrichs model). If $\varphi_{ \pm} \in \Phi_{ \pm}$, the expressions $\left\langle\varphi_{+} \mid E_{+}\right\rangle$and $\left\langle\varphi_{-} \mid E_{-}\right\rangle$are square integrable functions on the whole real axis, which are boundary values of functions analytic on the upper and lower half of the complex plane, respectively. In the context of Time Asymmetric Quantum Mechanics (TAQM) [32,42], these analytic functions are taken to be of Hardy class $[11,12]$. The action of $\psi^{D}$ on $\varphi_{+} \in \Phi_{+}$and $\psi^{G}$ on $\varphi_{-} \in \Phi_{-}$is given by the following integrals, respectively:

$$
\begin{aligned}
\left\langle\varphi_{+} \mid \psi^{D}\right\rangle & =\sqrt{\frac{\Gamma}{2 \pi}} \int_{-\infty}^{\infty} \frac{\left\langle\varphi_{+} \mid E_{+}\right\rangle d E}{\left(E-E_{R}\right)+i \Gamma / 2}, \\
\left\langle\varphi_{-} \mid \psi^{G}\right\rangle & =\sqrt{\frac{\Gamma}{2 \pi}} \int_{-\infty}^{\infty} \frac{\left\langle\varphi_{-} \mid E_{-}\right\rangle d E}{\left(E-E_{R}\right)-i \Gamma / 2}
\end{aligned}
$$

As a matter of fact, $\psi^{D}$ and $\psi^{G}$, represented as in (37), are normalizable vectors, although they lie outside of the domain of the total Hamiltonian $H$. This is a possible representation for Gamow vectors [11,40]. One quite interesting feature of Gamow vectors (37) is that they have an energy distribution of Breit-Wigner type. As is well known, this is a necessary and sufficient condition for a vector state to have an exponential non-decay probability [13].

However, the spectrum of $H$ has to be semibounded, so that we can assume, without lost of generality, that it covers the positive semiaxis $\mathbb{R}^{+} \equiv[0, \infty)$. Then (37-39) may not be the most adequate representation for Gamow vectors, as integrals on the variable energy go from $-\infty$ to $\infty$. Thus, let us replace (37) by the following expressions:

$$
\begin{aligned}
\left|f^{D}\right\rangle & =\sqrt{\frac{\Gamma}{2 \pi}} \int_{0}^{\infty} \frac{\left|E_{+}\right\rangle d E}{\left(E-E_{R}\right)+i \Gamma / 2}, \\
\left|f^{G}\right\rangle & =\sqrt{\frac{\Gamma}{2 \pi}} \int_{0}^{\infty} \frac{\left|E_{-}\right\rangle d E}{\left(E-E_{R}\right)-i \Gamma / 2} .
\end{aligned}
$$


We have replaced the integrals from $-\infty$ to $\infty$ by integrals from 0 to $\infty$, which is the range of the values of the energy for the continuum spectrum of the total Hamiltonian $H$. We call $\left|f^{D}\right\rangle$ and $\left|f^{G}\right\rangle$, the decaying and growing approximate Gamow vector (for the resonance given by the pole $z_{R}=E_{R}-i \Gamma / 2$ and its complex conjugate), respectively. These vectors are normalizable [41] and their norms are given by

$$
\left\|f^{D}\right\|^{2}=\left\|f^{G}\right\|^{2}=\frac{1}{\pi}\left(\frac{\pi}{2}+\arctan \left(\frac{2 E_{R}}{\Gamma}\right)\right) .
$$

Obviously,

$$
\lim _{2 E_{R} / \Gamma \mapsto \infty}\left\|f^{D}\right\|=\lim _{2 E_{R} / \Gamma \mapsto \infty}\left\|f^{G}\right\|=1 .
$$

The approximate Gamow vectors $\left|f^{D}\right\rangle$ and $\left|f^{G}\right\rangle$ have the following properties, which have been proven in [41]:

1. The spaces $\Phi_{ \pm}^{\times}$are endowed with a topology called the weak topology. In this topology, we have the following limit:

$$
\lim _{2 E_{R} / \Gamma \mapsto \infty} f^{D}=i(2 \pi \Gamma)^{1 / 2} \psi^{D}
$$

and a similar formula relates $f^{G}$ and $\psi^{G}$. Since all representations of the triplets $\Phi_{ \pm} \subset \mathcal{H} \subset \Phi_{ \pm}^{\times}$have equivalent topologies [11,12], this relation is independent on the representation of $\Phi_{ \pm}$(and hence of their duals $\Phi_{ \pm}^{\times}$).

2. Vectors $f^{D}$ and $f^{G}$ are not in the domain of the total Hamiltonian $H$.

3. In spite of the previous statement, we still can ask ourselves, if there is an average of the energy on $f^{D}$ and $f^{G}$. Although the answer is no, which can be deduced by observing that

$$
\left\langle f^{D}|H| f^{D}\right\rangle=\frac{E_{R}}{\pi} \int_{-2 E_{R} / \Gamma}^{\infty} \frac{d x}{1+x^{2}}+\frac{\Gamma}{2 \pi} \int_{-2 E_{R} / \Gamma}^{\infty} \frac{x d x}{1+x^{2}},
$$

where the second integral in (45) obviously diverges, we may take the limit as $2 E_{R} / \Gamma \longmapsto \infty$. Then, let us consider the Cauchy principal value on the resulting integrals in (45). This principal value is zero for the second integral and is $\pi$ for the former. Therefore, in the considered limit $\left\langle f^{D}|H| f^{D}\right\rangle \longmapsto E_{R}$, which coincides with the result given in [19]. Same result can be obtained for $f^{G}$.

4. The non-decay amplitude for $f^{D}$ (or $f^{G}$ ) can be easily obtained:

$$
\left\langle f^{D}\left|e^{-i t H}\right| f^{D}\right\rangle=\frac{\Gamma}{2 \pi} \int_{0}^{\infty} \frac{e^{-i t E_{R}} d E}{\left(E-E_{R}\right)^{2}+\Gamma^{2} / 4} .
$$

This integral is not a decreasing exponential on $t$. However, for either $E_{R}$ large (high resonance energies) or $\Gamma$ small (large lifetimes), the integral approaches to a decreasing exponential on time. In both cases, $2 E_{R} / \Gamma$ is high. Thus, deviations of the exponential law are less noticeable in these situations, a standard result [1]. 
Remark Assume that we have a RHS, $\Phi \subset \mathcal{H} \subset \Phi^{\times}$, and an eigenvalue equation in $\Phi^{\times}$of the form $H \psi^{D}=z_{R} \psi^{D}$. This properly means that for all $\varphi \in \Phi$, one has that $\left\langle H \varphi \mid \psi^{D}\right\rangle=z_{R}\left\langle\varphi \mid \psi^{D}\right\rangle$. Assume that we have a representation of the Gamow vector $\psi^{D}$ such that $\psi^{D}$ is in $\mathcal{H}$. Such a representation is possible $[11,12]$, but then $\psi^{D}$ cannot be in the domain of the Hamiltonian $H$ and, hence, $\psi^{D}$ cannot belong to $\Phi$, as $\Phi$ should always be contained in the domain of $H[11,12]$. Then, consider $\left\langle\psi^{D}|H| \psi^{D}\right\rangle$. For any representation such that $\psi^{D}$ be normalizable we can say: (i) the product $\left\langle\psi^{D}|H| \psi^{D}\right\rangle$ does not make sense in Hilbert space, since $\psi^{D}$ is not in the domain of $H$; (ii) Also, $\left\langle\psi^{D}|H| \psi^{D}\right\rangle$ does not make sense in the RHS $\Phi \subset \mathcal{H} \subset \Phi^{\times}$, since $\psi^{D}$ is not in $\Phi$. Consequently, an expression like $\left\langle\psi^{D}|H| \psi^{D}\right\rangle=z_{R}\left\langle\psi^{D} \mid \psi^{D}\right\rangle$ is now wrong even if, as is the case here, the scalar product $\left\langle\psi^{D} \mid \psi^{D}\right\rangle$ is well defined. In addition, the identity $\left\langle\psi^{D}|H| \psi^{D}\right\rangle=E_{R}$ makes sense only as a limit, precisely as we have explained in the paragraph numbered 3 right above.

\section{Are Gamow States Pure States?}

Pure states are given by normalizable vectors in a Hilbert space. However, Gamow vectors admit a representation as functionals which are either non-normalizable or if they are normalizable, they must range outside the domain of the total Hamiltonian $H$. The representation of Gamow states as functionals is indeed a representation of them as vector states, so that one is tempted to consider Gamow states as pure states. However, pure states preserve norms with time evolution and Gamow states decay (or grow) exponentially. The scenario here is somehow confusing. We need a formalism which discriminates without doubt pure states of other possible states which are not pure, either mixtures or something else.

In the connection with some problems of statistical mechanics, in particular to include the so called states with singular diagonal [25-27], Antoniou et al. [24] have developed an algebraic formalism for the description of states and observables. In this formalism, we can give a precise meaning to Gamow states. We begin with the Hamiltonian pair $\left\{H_{0}, H=H_{0}+V\right\}$. For simplicity, it is often convenient to assume that both have a non-degenerate absolutely continuous spectrum, which is $[0, \infty)$. Then, $H_{0}$ admits the following spectral decomposition:

$$
H_{0}=\int_{0}^{\infty} E|E\rangle\langle E| d E
$$

where $|E\rangle$ are the generalized eigenvectors of $H_{0}$ with eigenvalue $E$, i.e., $H_{0}|E\rangle=$ $E|E\rangle$. We say that an operator $O$ is compatible with $H_{0}$ if it has the form:

$$
O=\int_{0}^{\infty} d E O_{E}|E\rangle\left\langle E\left|+\int_{0}^{\infty} d E \int_{0}^{\infty} d E^{\prime} O_{E E^{\prime}}\right| E\right\rangle\left\langle E^{\prime}\right|,
$$

where $O_{E}$ is a function of the variable $E$ and $O_{E E^{\prime}}$ is a function of the variables $E$ and $E^{\prime}$.

Assume now that the Møller operators $\Omega_{ \pm}$exists and are asymptotically complete. Here $\Omega_{-}$represents the incoming and $\Omega_{+}$the outgoing wave operator. Then, $H=$ 
$\Omega_{ \pm} H_{0} \Omega_{ \pm}$(at least for the restrictions of $H_{0}$ and $H$ to their respective absolutely continuous subspaces). The generalized eigenvectors of $H$ can be defined as $\left|E_{ \pm}\right\rangle=$ $\Omega_{ \pm}|E\rangle[12]$. Then, for any operator $O$ compatible with $H_{0}$, let us define:

$$
O_{ \pm}:=\Omega_{ \pm} O \Omega_{ \pm}=\int_{0}^{\infty} d E O_{E}\left|E_{ \pm}\right\rangle\left\langle E_{ \pm}\left|+\int_{0}^{\infty} d E \int_{0}^{\infty} d E^{\prime} O_{E E^{\prime}}\right| E_{ \pm}\right\rangle\left\langle E^{\prime}{ }_{ \pm}\right|
$$

We say that these operators are compatible with $H$. Operators compatible with $H$ form a pair of involutive algebras $\mathcal{A}_{ \pm}[24,28,29]$ with identity. The involution is given by $\left(O_{ \pm} \in \mathcal{A}_{ \pm}\right)$

$$
O_{ \pm} \longmapsto O_{ \pm}^{\dagger}:=\int_{0}^{\infty} d E O_{E}^{*}\left|E_{ \pm}\right\rangle\left\langle E_{ \pm}\left|+\int_{0}^{\infty} d E^{\prime} \int_{0}^{\infty} d E O_{E^{\prime} E}^{*}\right| E^{\prime}{ }_{ \pm}\right\rangle\left\langle E_{ \pm}\right|
$$

where the star denotes complex conjugation. Operators verifying $O_{ \pm}=O_{ \pm}^{\dagger}$ are called observables. In order to simplify the above notation, let us write $\left.\mid E_{ \pm}\right):=\left|E_{ \pm}\right\rangle\left\langle E_{ \pm}\right|$ and $\left.\mid E E_{ \pm}^{\prime}\right):=\left|E_{ \pm}\right\rangle\left\langle E_{ \pm}^{\prime}\right|$. Then, (49) can be rewritten as:

$$
\left.\left.O_{ \pm}=\int_{0}^{\infty} d E O_{E} \mid E_{ \pm}\right)+\int_{0}^{\infty} d E \int_{0}^{\infty} d E^{\prime} O_{E E^{\prime}} \mid E E^{\prime}{ }_{ \pm}\right)
$$

The respective identities $I_{ \pm}$in $\mathcal{A}_{ \pm}$take the form:

$$
I_{ \pm}=\int_{0}^{\infty} d E\left|E_{ \pm}\right\rangle\left\langle E_{ \pm}\left|=\int_{0}^{\infty} d E\right| E_{ \pm}\right)
$$

Let us consider the linear space of linear mappings (also called functionals) $\rho_{ \pm}$ from $\mathcal{A}_{ \pm}$into the set of complex numbers $\mathbb{C}$. The action of $\rho_{ \pm}$into $O_{ \pm} \in \mathcal{A}_{ \pm}$is denoted as $\left(\rho_{ \pm} \mid O_{ \pm}\right)$. A state on $\mathcal{A}_{ \pm}$is usually defined as a linear mapping $\rho_{ \pm}$on $O_{ \pm} \in \mathcal{A}_{ \pm}$verifying the following conditions: (i) Positivity: $\left(\rho_{ \pm} \mid O_{ \pm}{ }^{\dagger} O_{ \pm}\right) \geq 0$. (ii) Normalization: $\left(\rho_{ \pm} \mid I_{ \pm}\right)=1$.

A typical functional on $\mathcal{A}_{ \pm}$is written in our notation as [28,29]

$$
\rho_{ \pm}=\int_{0}^{\infty} d E \rho_{E}\left(E_{ \pm} \mid+\int_{0}^{\infty} d E \int_{0}^{\infty} d E^{\prime} \rho_{E E^{\prime}}\left(E E^{\prime}{ }_{ \pm}\right.\right.
$$

Here, $\rho_{E}$ and $\rho_{E} E^{\prime}$ are functions or distributions on the considered variables. If they are distributions, $O_{E}$ and $O_{E E^{\prime}}$ must belong to suitable spaces of test functions. Then [28,29], the action of $\rho_{ \pm}$into $O_{ \pm} \in \mathcal{A}_{ \pm}$is written as

$$
\left(\rho_{ \pm} \mid O_{ \pm}\right)=\int_{0}^{\infty} d E \rho_{E} O_{E}+\int_{0}^{\infty} d E \int_{0}^{\infty} d E^{\prime} \rho_{E E^{\prime}} O_{E E^{\prime}}
$$

where we have used the following identities:

$$
\left(E_{ \pm} \mid E^{\prime}{ }_{ \pm}\right)=\delta\left(E-E^{\prime}\right), \quad\left(E E^{\prime}{ }_{ \pm} \mid w w_{ \pm}^{\prime}\right)=\delta(E-w) \delta\left(E^{\prime}-w^{\prime}\right)
$$


which are a direct consequence of $\left\langle E_{ \pm} \mid E_{ \pm}^{\prime}\right\rangle=\delta\left(E-E^{\prime}\right)$.

In this formalism, Antoniou et al. [24] classify three types of states (see also [28, 29]):

(i) Pure states In this representation, a state $\psi$ is pure if and only if there exists a function $\psi(E)$ of the energy $E$ such that $\rho_{E}=|\psi(E)|^{2}$ and $\rho_{E E^{\prime}}=\psi^{*}(E) \psi\left(E^{\prime}\right)$, where the star denotes complex conjugation.

(ii) Mixtures If $\rho$ is a mixture of pure states, then, there exists a spectral decomposition of the type $\rho=\sum_{i} \lambda_{i}\left|\psi_{i}\right\rangle\left\langle\psi_{i}\right|$, where $\left|\psi_{i}\right\rangle$ are pure states. Assume now that, in the energy representation, $\left|\psi_{i}\right\rangle$ can be represented as a function $\psi_{i}(E)$. Then, $\rho_{E}=\sum_{i}\left|\psi_{i}(E)\right|^{2}$ and $\rho_{E E^{\prime}}=\sum_{i} \lambda_{i} \psi_{i}^{*}(E) \psi_{i}\left(E^{\prime}\right)$. Then, the state $\rho$ is a mixture if and only if it is not a pure state and $\rho_{E E}=\rho_{E}$.

(iii) Singular diagonal states These states were introduced in the context of quantum systems far from equilibrium [25-27]. As a matter of fact, the above formalism was introduced in [24] order to accommodate this kind of states. We are not discuss their properties here, but the interested reader could go to [24]. Here, we simply define these states as those having the property that $\rho_{E E} \neq \rho_{E}$.

All these types of states have the properties of positivity and normalization.

Gamow vectors can be represented as functionals in this formalism, although not in a unique way. As a matter of fact, we have found three different possible ways to define a Gamow state as a functional over the algebras $\mathcal{A}_{ \pm}$. In order to do this, we need to define the algebras $\mathcal{A}_{ \pm}$so that the functions $O_{E}$ can be analytically continued to a half plane as functions of a complex variable. A similar construction should go for $O_{E E^{\prime}}$, see [29] for details. In this case, $\delta_{z}$ would be the functional that maps $O_{E}$ into its value at the point $z, O_{z}$. Analogously, $\delta_{z} \otimes \delta_{z^{\prime}}$ maps $O_{E E^{\prime}}$ into $O_{z z^{\prime}}$.

Now, we are in the position to define a Gamow states in this context. In the sequel, we shall discuss with some detail a first construction and leave for the next subsection the other two. Proofs of their properties are the same in all cases. Then, let us define the following functional over $\mathcal{A}_{+}$:

$$
\rho_{D}=\int_{0}^{\infty} d E \int_{0}^{\infty} d E^{\prime} \delta_{z_{R}^{*}} \otimes \delta_{z_{R}}\left(E E_{+}^{\prime} \mid\right.
$$

where $z_{R}=E_{R}-i \Gamma / 2$ is the resonance pole. In addition, there exists a functional, $\rho_{G}$, on $\mathcal{A}_{-}$for the growing Gamow vector and it is given by

$$
\rho_{G}=\int_{0}^{\infty} d E \int_{0}^{\infty} d E^{\prime} \delta_{z_{R}} \otimes \delta_{z_{R}^{*}}\left(E E_{-}^{\prime} \mid .\right.
$$

Henceforth, we call $\rho_{D}$ and $\rho_{G}$ the decaying Gamow functional and the growing Gamow functional, respectively. They have the following properties [29]:

(i) These operators have trace zero:

$$
\operatorname{tr} \rho_{D}=\left(\rho_{D} \mid I_{+}\right)=0, \quad \operatorname{tr} \rho_{G}=\left(\rho_{G} \mid I_{-}\right)=0
$$

and therefore, they cannot fit into the standard definition of state. 
(ii) Both Gamow functionals are positive, which means that for any observable $O_{+} \in$ $\mathcal{A}_{+}$or $O_{-} \in \mathcal{A}_{-}$, one has $\left(\rho_{D} \mid O_{+}{ }^{\dagger} O_{+}\right) \geq 0$ and $\left(\rho_{G} \mid O_{-}{ }^{\dagger} O_{-}\right) \geq 0$.

(iii) All the energy momenta with respect either to $\rho_{D}$ or $\rho_{G}$ vanish:

$$
\left(\rho_{D} \mid H^{n}\right)=\left(\rho_{G} \mid H^{n}\right)=0, \quad n=0,1,2, \ldots,
$$

where, according to (44) and (46) one has

$$
H^{n}=\int_{0}^{\infty} d E E^{n}\left|E_{ \pm}\right\rangle\left\langle E_{ \pm}\left|=\int_{0}^{\infty} d E E^{n}\right| E_{ \pm}\right), \quad n=0,1,2 \ldots
$$

For $n=1$, we have that (59) is in agreement with (30), which states that the mean value of the energy for a Gamow state should be zero.

(iv) The functional $\rho_{D}$ decays for $t>0$ and the functional $\rho_{G}$ grows for $t<0$, as $t \longmapsto 0,[28,29]$ :

$\left(\rho_{D}(t) \mid O_{+}\right)=e^{-\Gamma t}\left(\rho_{D} \mid O_{+}\right), \quad t>0 ; \quad\left(\rho_{G}(t) \mid O_{-}\right)=e^{\Gamma t}\left(\rho_{G} \mid O_{-}\right), \quad t<0$,

for arbitrary operators $O_{ \pm} \in \mathcal{A}_{ \pm}$. These decay modes can be found if we use Hardy functions on a half plane in order to define $O_{E}$ and $O_{E E^{\prime}}[28,29]$. Using entire functions instead, we can show that time behavior (15) can be extended for all values of $t$ [39].

(v) The functionals $\rho_{D}$ and $\rho_{G}$ are time reversal of each other.

These representations of Gamow states as functionals are well defined and satisfy requisites like a correct time behavior and the property that $\rho_{D}$ and $\rho_{G}$ are the time reversal of each other. The energy averages for these states are zero as suggested in $[4,20]$. The fact that both $\rho_{D}$ and $\rho_{G}$ have zero trace, implies that they do not fit with the standard definition of state.

\subsection{A Second Definition for the Gamow Functionals}

The definition for the Gamow functionals given in the previous section is not very satisfactory for two reasons: First of all, their trace is equal to zero (non-normalizability), so that they should not be considered as states. Secondly, all the moments of the energy are equal to zero, not only the energy average, see (59). Here, we propose two other possible definitions that fix both related problems. In the first one, the mean value of the energy is $z_{R}=E_{R}-i \Gamma / 2$, for $\rho_{D}$ and its complex conjugate for $\rho_{G}$, and in the second is equal to the resonance energy $E_{R}$, as suggested in [19]. Then, we propose the following two alternative definitions for the decaying Gamow functional:

$$
\rho_{D}=\int_{0}^{\infty} d E \delta_{z_{R}}\left(E_{+} \mid+\int_{0}^{\infty} d E \int_{0}^{\infty} d E^{\prime} \delta_{z_{R}^{*}} \otimes \delta_{z_{R}}\left(E, E_{+}^{\prime} \mid\right.\right.
$$

and

$$
\rho_{D}:=\int_{0}^{\infty} d E \delta_{E_{R}}\left(E_{+} \mid+\int_{0}^{\infty} d E \int_{0}^{\infty} d E^{\prime} \delta_{z_{R}^{*}} \otimes \delta_{z_{R}}\left(E, E_{+}^{\prime} \mid\right.\right.
$$


Similar formulas will define the growing Gamow functional, $\rho_{G}$. Both, (62) and (63) have trace one:

$$
\left(\rho_{D} \mid I_{+}\right)=\left(\rho_{G} \mid I_{-}\right)=1,
$$

and and both are positive. Therefore, both functionals (62) and (63) are states. However, both functionals (62) and (63) do not satisfy the condition to be a pure state. Furthermore, for the particular choice of $\rho_{E}$ and $\rho_{E E^{\prime}}$ in (62) and in (63), they cannot be even a mixture. Therefore, they are singular diagonal states.

With respect the momenta of the energy, with (62), one has:

$$
\left(\rho_{D} \mid H\right)=z_{R}, \quad\left(\rho_{D} \mid H^{n}\right)=z_{R}^{n}, \quad n=0,1,2, \ldots,
$$

while for (63), we have:

$$
\left(\rho_{D} \mid H\right)=E_{R}, \quad\left(\rho_{D} \mid H^{n}\right)=E_{R}^{n}, \quad n=0,1,2, \ldots
$$

Concerning the time evolution, observe that for both definitions (62) and (63), $\rho_{D}$ is decomposed in a sum of two terms. Let us write $\rho_{D}=\rho_{R}+\rho_{S}$, where $R$ and $S$ stand for regular and singular, respectively. It can be easily shown that the regular term does not evolve, while the singular term, which coincides with (56), decays exponentially, so that

$$
\rho_{D}(t)=\rho_{R}(0)+e^{-\Gamma t} \rho_{S}(0)
$$

and a similar formula for $\rho_{G}(t)=\rho_{R}(0)+e^{\Gamma t} \rho_{S}(0)$.

The conclusion is that Gamow states can be described as functionals over an algebra including observables, at least in three different ways. They give different energy averages depending on the definition used: either zero, $z_{R}=E_{R}-i \Gamma / 2$ or $E_{R}$. The choice giving zero mean value of the energy does not satisfy the definition of state, contrary to the other two. None of the three choices represents a pure state nor a mixture.

\section{Concluding Remarks}

Along the present paper, we discuss some properties of Gamow vectors, which are vector states for the exponentially decaying (or its counterpart and time reversal image, the exponentially growing) contribution to the unstable quantum states. These Gamow states provide a good picture of a quantum unstable state during a large time interval. However, Gamow vectors do not admit a scalar product normalization as they do not belong to the Hilbert space of pure states of the system under consideration. In most cases, we cannot even define brackets among Gamow vectors as we can do, for instance, among plane waves.

These facts produce difficulties in the treatment of unstable quantum states using Gamow vectors as vector states. For instance, serious problems arise when one needs to define energy averages on Gamow states. As we have seen, there are different approaches to define these averages. As a matter of fact, there is no agreement in the 
scientific community on what the energy of a Gamow state should be. Even worse, many of the proposed approaches are ill defined.

Here, we use the Friedrichs model as a working example to illustrate some of these difficulties.

The effect of the background on the decay is small or even negligible for times neither too small nor too large. This allows to ignore it for a wide time interval and use a description of quantum unstable states based solely on the space of Gamow vectors. In this case, we can easily define brackets allowing to define energy averages for Gamow states formally consistent.

The standard theory of Gamow states shows that there exists normalizable state vectors arbitrarily close, in some sense, to Gamow states. The task of finding such approximate normalizable vectors is often difficult. Here, we propose a way to construct an approximation of this kind using the energy representation and discuss its accuracy. This has been used to conclude that the energy average on a Gamow state should coincide with the resonance energy $E_{R}$. This is possibly the best choice for this average.

In principle, the question whether a Gamow state is a pure state or not is not trivial. The solution of this problem may have some consequences, as for instance if the entropy of a Gamow state should be zero or not. The question of the purity of a Gamow state can be solved unambiguously. In fact, Gamow states can be defined as functional on algebras of observables. Here, we have proposed three different forms of defining these functionals. None of them satisfies the properties required to pure states. Furthermore, energy averages are well defined in all cases, although our three different definitions give three different values for these averages. Nevertheless, these values coincide with those given on some of the approaches discussed in the literature.

\section{References}

1. Fonda, L., Ghirardi, G.C., Rimini, A.: Decay theory of unstable quantum systems. Rep. Progr. Phys. 41, 588-631 (1978)

2. Fischer, M.C., Gutierrez-Medina, B., Raizen, M.G.: Observation of the quantum Zeno and anti-Zeno effects in an unstable system. Phys. Rev. Lett. 87, 040402 (2001)

3. Rothe, C., Hintschich, S.L., Monkman, A.P.: Violation of the exponential-decay law at long times. Phys. Rev. Lett. 96, 163601 (2006)

4. Nakanishi, N.: A theory of clothed unstable particles. Progr. Theor. Phys. 19, 607-621 (1958)

5. Bohm, A.: The Rigged Hilbert Space and Quantum Mechanics, Springer Lecture Notes in Physics, vol. 78. Springer, New York (1978)

6. Gelfand, I.M., Vilenkin, N.Y.: Generalized Functions: Applications of Harmonic Analysis. Academic, New York (1964)

7. Roberts, J.E.: Rigged Hilbert spaces in quantum mechanics. Commun. Math. Phys. 3, 98-119 (1966)

8. Antoine, J.P.: Dirac formalism and symmetry problems in quantum mechanics: General Dirac formalism. J. Math. Phys. 10, 53-69 (1969)

9. Melsheimer, O.: Rigged Hilbert spaces as an extended mathematical formalism for quantum mechanics: 1 General theory. J. Math. Phys. 15, 902-916 (1974)

10. Bohm, A.: Resonance poles and Gamow vectors in the rigged Hilbert space formulation of quantum mechanics. J. Math. Phys. 22, 2813-2823 (1981)

11. Bohm, A., Gadella, M.: Dirac Kets, Gamow Vectors and Gelfand Triplets, Springer Lecture Notes in Physics, vol. 348. Springer Verlag, Berlin (1989)

12. Civitarese, O., Gadella, M.: Physical and mathematical aspects of Gamow states. Phys. Rep. 396, 41-113 (2004) 
13. Bohm, A.: Quantum Mechanics. Foundations and Applications, 3rd edn. Springer, New York (2001)

14. Julve, J., de Urriés, F.J.: Inner products of resonance solutions in 1D quantum barriers. J. Phys. A 43, $175301(2010)$

15. Julve, J., Turrini, S., de Urriés, F.J.: Inner products of energy eigenstates for a 1-D quantum barrier. Int. J. Theor. Phys. 53, 971-984 (2014)

16. Castagnino, M., Gadella, M., Gaioli, F., Laura, R.: Gamow vectors and time assymmetry. Int. J. Theor. Phys. 38, 2823-2865 (1999)

17. Berggren, T.: On the use of resonant states in eigenfunction expansions of scattering and reaction amplitudes. Nucl. Phys. A 109, 265-287 (1968)

18. Berggren, T.: Expectation value of an operator in a resonant state. Phys. Lett. B 373, 1-4 (1996)

19. Civitarese, O., Gadella, M., Id Betan, R.: On the mean value of the energy for resonance states. Nucl. Phys. A 660, 255-266 (1999)

20. Petrosky, T., Prigogine, I., Tasaki, S.: Quantum theory of non-integrable systems. Phys. A 173, 175-242 (1991)

21. Bollini, C.G., Civitarese, O., de Paoli, A.L., Rocca, M.C.: Gamow states as continuous linear functionals over analytical test functions. J. Math. Phys. 37, 4235-4242 (1996)

22. Exner, P.: Open Quantum Systems and Feynman Integrals. Reidel, Dordrecht (1984)

23. Reed, M., Simon, B.: Analysis of Operators. Academic, New York (1978)

24. Antoniou, I.E., Laura, R., Suchanecki, Z., Tasaki, S.: Intrinsic irreversibility of quantum systems with diagonal singularity. Phys. A 241, 737-772 (1997)

25. van Hove, L.: Energy corrections and persistent perturbation effects in continuous spectra. Physica 21, 901 (1955)

26. van Hove, L.: The approach to equilibrium in quantum statistics. Physica 23, 441 (1957)

27. van Hove, L.: The ergodic behaviour of quantum many-body systems. Physica 25, 268 (1959)

28. Castagnino, M., Gadella, M., Id Betan, R., Laura, R.: The Gamow functional. Phys. Lett. A 282, 245-250 (2001)

29. Castagnino, M., Gadella, M., Id Betan, R., Laura, R.: Gamow functionals on operator algebras. J. Phys. A 34, 10067-10083 (2001)

30. Friedrichs, K.O.: On the perturbation of continuous spectra. Commun. Appl. Math. 1, 361-406 (1948)

31. Gadella, M., Pronko, G.P.: The Friedrichs model and its use in resonance phenomena. Fortschr. Phys. 59, 795-859 (2011)

32. Bohm, A., Gadella, M., Kielanowski, P.: Time asymmetric quantum mechanics. SIGMA 7, 086 (2011)

33. Bohm, A.R., Erman, F., Uncu, H.: Resonance phenomena and time asymmetric quantum mechanics. Turk. J. Phys. 35, 209-240 (2011)

34. Antoniou, I., Dmitrieva, L., Kuperin, Y., Melnikov, Y.: Resonances and the extension of dynamics to rigged Hilbert space. Comput. Math. Appl. 34, 399-425 (1997)

35. Civitarese, O., Gadella, M.: On the concept of entropy for decaying states. Found. Phys. 43, 1275-1294 (2013)

36. Antoniou, I., Pronko, G.P., Karpov, E., Yarevsky, E.: Oscillating decay of an unstable system. Int. J. Theor. Phys. 42, 2403-2421 (2003)

37. Kukulin, V.I., Krasnapolsky, V.M., Horacek, J.: Theory of Resonances. Principles and Applications. Academia, Praha (1989)

38. Gyarmati, B., Vertse, T.: On the normalization of Gamow functions. Nucl. Phys. A 160, 523-528 (1971)

39. Gadella, M., Laura, R.: Gamow dyads and expectation values. Int. J. Quantum Chem. 81, 307-320 (2001)

40. Bohm, A.: Resonance poles and Gamow vectors in the rigged Hilbert space formulation of quantum mechanics. J Math. Phys. 21, 2813-2823 (1981)

41. Gadella, M.: Gamow vectors: miscellaneous results. J. Phys. Conf. Ser. 128, 012038 (2008)

42. Bohm, A.R., Loewe, M., Van de Ven, B.: Time asymmetric quantum theory - I. Modifying an axiom of quantum physics. Fortschr. Phys. 51, 551-568 (2003) 